\title{
MqsA antitoxin degradation is regulated by zinc occupancy and oxidation Margaret Vos ${ }^{1}$, Benjamin Piraino ${ }^{2}$, Christopher LaBreck ${ }^{3}$, Negar Rahmani ${ }^{4}$, Catherine Trebino ${ }^{5}$, Marta Schoenle ${ }^{6}$, Wolfgang Peti $^{7}$, Jodi Camberg ${ }^{8}$, Rebecca Page ${ }^{9}$ \\ ${ }^{1}$ University of Connecticut Health Center ${ }^{2}$ University of Rhode Island, ${ }^{3}$ University of Rhode Island, ${ }^{4}$ University of Rhode Island, ${ }^{5}$ University of Rhode Island, ${ }^{6}$ University of Arizona, ${ }^{7}$ University of Connecticut Health Center, ${ }^{8}$ University of Rhode Island, ${ }^{9}$ University of Connecticut Health Center, vos@uchc.edu
}

While it is well established that antitoxins of toxin-antitoxin (TA) systems are selectively degraded by bacterial proteases in response to stress, the mechanism leading to selective degradation of specific antitoxins is unknown. We investigated this mechanism using reconstituted Escherichia coli ClpXP proteolytic machinery in vitro to monitor degradation of MqsA, the antitoxin component of the MqsRA TA system. MqsA is a ClpXP proteolysis substrate, and degradation is regulated by zinc occupancy in MqsA and MqsR toxin binding. Using NMR chemical shift perturbation mapping, we show that MqsA is targeted directly to ClpXP via the ClpX substrate targeting Ndomain, and ClpX mutations that disrupt $\mathrm{N}$-domain binding inhibit $\mathrm{ClpXP}$ mediated degradation. We also discovered that MqsA contains a cryptic N-domain recognition sequence that is accessible only in the absence of zinc and MqsR toxin, both of which stabilize the MqsA fold. This recognition sequence is transplantable and sufficient to target a fusion protein for degradation. Furthermore, crystallographic data of MqsA homologs show the critical nature of zinc in stabilizing the protein. Based on our data, we propose a model in which oxidative stress selectively targets nascent, zinc-free MqsA, resulting in rapid oxidation of the zinc-coordinating cysteine residues and exposure of the $\mathrm{ClpX}$ recognition motif for ClpXP mediated degradation. 